\title{
Effect of Different Dietary Levels of Protein on the Proximate Composition of Genetically Improved Farmed Tilapia (GIFT) from Pakistan
}

\author{
Anila Kousar, Muhammad Naeem* and Samrah Masud
}

Institute of Pure and Applied Biology, Zoology Division, Bahauddin Zakariya University, Multan, 60800, Pakistan.

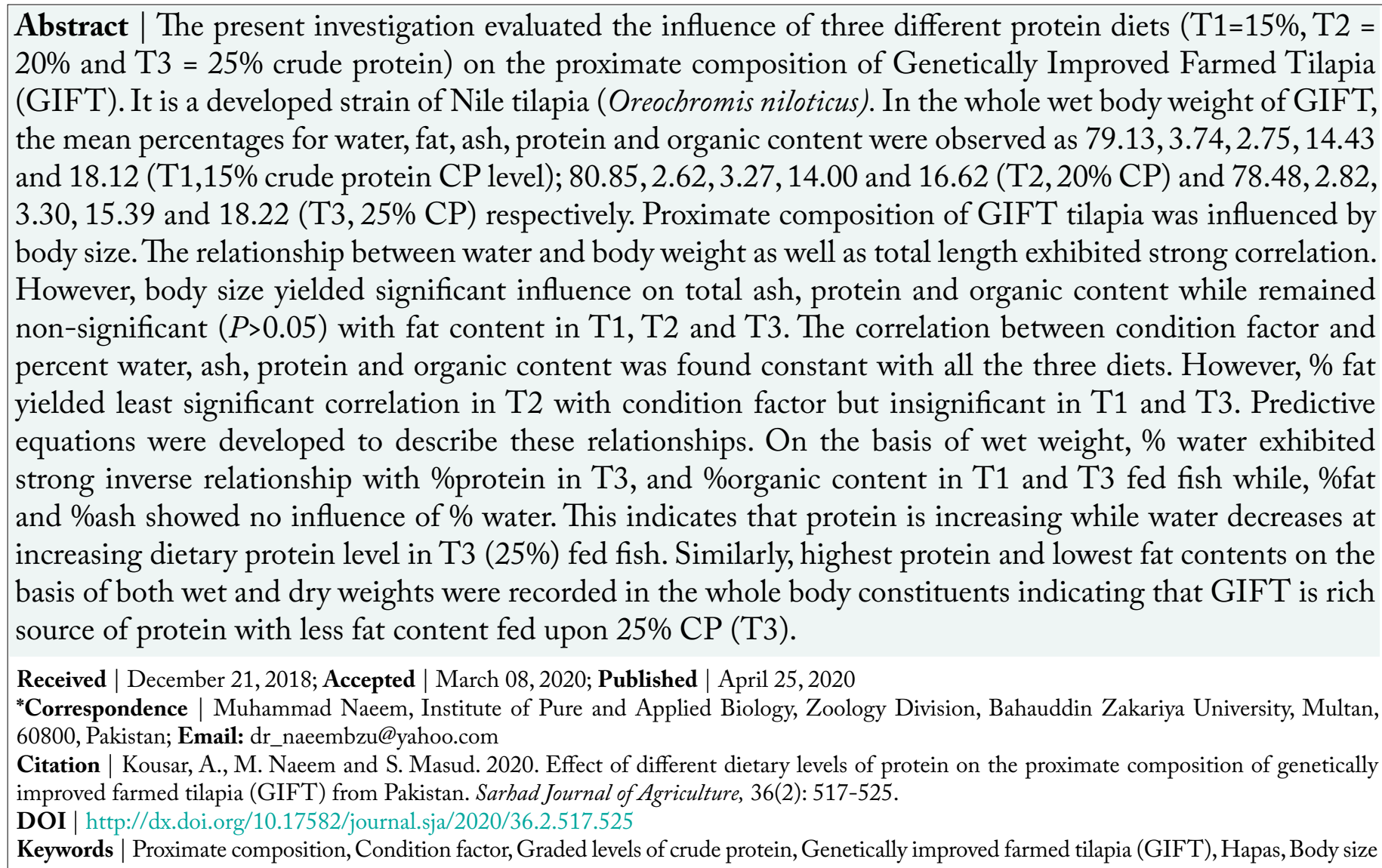

\section{Introduction}

$\mathrm{I}$ is important for any food to contain quality nutrients (Nettleton, 1992). According to James (1998) fish is consumed by a large number of people in the whole world and provides quality nutrients to their consumers. The quality of food depends on the quantities of nutrients present in it and their bioavailability. Man is interested in healthy food because health is dependent on quality diet (Lie, 2001). Fish is a good source of protein and fish fat is important because it contains n-3 fatty acids (Bennett et al., 2018). It is important to study the status of nutrients present in fish due to high demand of fish and its products (Adefemi, 2011). The quality of nutrients present in food describes its proximate composition. It is necessary to assess the nutritional value and quality of fish being eaten (Azam et al., 2004). 
There is an increasing interest in the studies of body composition of fish because safety and quality of fish and its products are in great demand (Dumas et al., 2010). The body composition represents an important feature of nutrients quality. It can be assessed through the analysis of protein, fat and ash present in the food making it valuable (Shehawy et al., 2016).

Fish is consumed by large human population globally due to presence of high quality protein, its availability, palatability and deliciousness (Foran et al., 2005). So, it is necessary to check the proximate composition of fish before its consumption (Fawole et al., 2007) as it promotes growth (Ugwu et al., 2007). Bano et al. (2019) documented that increasing level of protein in diet enhances the whole body contents of protein.

Nile tilapia (Oreochromis niloticus) is a popular fish (Jim et al., 2017) and shows variations in protein requirement with the size/age, protein quality, water temperature, salinity, non-protein energy levels, presence of natural food and allowance of feed (NRC, 1993). A selective breeding program was carried out to improve the Nile tilapia. A base population of Nile tilapia was selected for several generations to develop the GIFT strain (Eknath and Acosta, 1998). The GIFT strain was developed from 20 years of selection (Bentsen et al., 1998).

Available data on proximate analysis of GIFT is scarce (De-Silva et al., 2016). So, the present study aimed to investigate the impact of different protein diets on proximate composition of GIFT.

\section{Materials and Methods}

Three diets consisting of $15 \%, 20 \%$ and $25 \%$ crude protein were prepared using the cheap plant ingredients. Plant origin feed ingredients used to formulate experimental feed included soyabean meal, sunflower meal, canola meal, rice polish, mustard meal, wheat bran, corn gluten and limited quantities of fish meal. These ingredients were purchased from the local market and were grinded finely for its easy ingestion by fish.

Five days old GIFT fingerlings of were collected, acclimatized for two weeks, and transferred to hapas $(8 \times 6 \times 3 \mathrm{ft})$ at random. Fingerlings were fed fish meal @ $10 \%$ during acclimatization period and experimental feed at the rate of $5 \%$ up to the end of experiment.
The experiment was carried out in duplicate for ninety days. All the specimens were collected on the completion of feeding trial from the fish farm (Tawakkal Tilapia Hatchery) where experiment was carried out. It is located at Tawakkal Nagar Muzaffar Garh, Punjab, Pakistan. Ten fishes were randomly selected from each treatment hapa for the estimation of biochemical constituents of studied GIFT. These samples were washed, dried and MS222 was used for anaesthetizing them. For the measurement of wet weight and total length of samples digital electric balance (Shimadzu ELB - 300 Japan) and measuring wooden tray were used respectively.

All the dead and pre- weighed samples were placed individually in an aluminum foil tray. These trays were kept in an electric oven at $70^{\circ} \mathrm{C}$ till constant weight of samples was obtained. Percent dry weight and water contents in fish were measured as given below.

$$
\% \text { dry weight }=(\text { dry weight } / \text { wet weight }) \times 100
$$

All the samples were then grinded using pestle and mortar to a fine powder for analyzing other body components.

Fat content in samples were measured with the help of dry extraction method (Bligh and Dyer, 1959). In this method, chloroform and methanol were mixed together in a ratio of 1:2. One gram of fish powder was taken in a clean glass test tube and $10 \mathrm{ml}$ of this mixture was poured in it. Test tubes were stirred well and left for one night. Clean small glass tubes were used for taking the clear supernatant from the test tubes. In the incubator (Memmert ${ }^{\circledR} 8540$ ) all bottles were kept for evaporation of samples at $50^{\circ} \mathrm{C}$ to obtain fat. The obtained contents of fat were measured using electric balance Shimadzu ELB-300 Japan. The amount of fat was then obtained by the subtraction of weight of empty glass bottles from the fat containing bottles. Percent fat in all the samples was calculated as given below.

Percent fat in the wet weight of fish $=($ total fat present in fish / wet weight of the fish body) $\times 100$

Percent fat present in dry weight $=($ total amount of fat in fish / dry weight of fish) $\times 100$

The amount of ash in samples was measured by taking one gram powder fish in china crucible which were pre weighed. These crucibles were then kept in muffle 
furnace for 24 hours at a temperature of $500^{\circ} \mathrm{C}$. After removing from furnace weight of crucibles was taken again on cooling. Weight of ash was obtained on subtracting the content of ash from the weight of initial sample. Same method was used for getting percent ash in dry and wet weights of fish.

The mass of ash and fat was subtracted from the dry weight of fish to get total amount of protein by following Caulton and Bursell (1977) and Salam and Davies (1994). Amount of percent protein in wet and dry fish weight was obtained similar to $\%$ ash and $\%$ fat.

The condition factor was measured by dividing the weight of fish with the cube of length of fish and then multiplying with 100 following Wooton (1990) and Salam and Davies (1994).

\section{Analysis of data}

Means, ranges, standard deviations and errors were calculated. MS-Excel program was used to apply regression, correlation coefficients and student's t- test following Zar (1996) and Naeem and Ishtiaq (2011).

\section{Results and Discussion}

Effect of three different protein diets ( $\mathrm{T} 1=15 \% \mathrm{CP}$ $\mathrm{T}=20 \% \mathrm{CP}$ and $\mathrm{T} 3=25 \% \mathrm{CP}$ ) in GIFT was evaluated for proximate composition. Table 1 summarizes means of percent water, ash, fat, protein and organic content in the dry and wet weights of fish for T1, T2 and $\mathrm{T} 3$ are summarized in Table 1.

Water content in GIFT were found highest in treatment 2 (T2), in which fish were fed with $20 \%$ dietary protein, followed by treatment 1 (T1, 15\% dietary protein) and treatment 3 (T3, 25\% dietary protein). Protein and organic contents were highest while fat was lowest in T3 fish on dry weight basis. Ash content was higher in $\mathrm{T} 2$ fed fish than $\mathrm{T} 1$ and T3 (Table 1).

Most of the \% body constituents yielded nonsignificant correlation $(P>0.05)$ with \% water except for \%fat dry weight (T2, $P<0.01), \%$ protein wet weight (T3, $P<0.001)$ and $\%$ organic content wet weight (T1and T3, $P<0.001)$ and $(\mathrm{T} 2, P<0.05)$ (Table 2).

The regression analysis yielded positive and strong correlation $(P<0.001)$ for $\log$ transformed data of total water with wet body weight (Table 3 ) and total length (Table 4) for all the treatments, while, correlation of total fat was found non-significant $(P>0.05)$ for all treatments. $\log$ transformed data of total protein and total ash indicated positive and significant correlations with body weight and total length in GIFT fed $15 \%$ and $25 \% \mathrm{CP}$ but relationship was found nonsignificant $(P>0.05)$ for $20 \% \mathrm{CP}$ fed fish. Organic content yielded significant positive correlation for all the three treatments. The value of slope $(b<3)$ exhibited negative allometry in all the parameters of proximate composition for T1, T2 and T3 with total length (Table 4). Negative allometry $(b<1)$ was found in $\log$ water $(\mathrm{T} 1, \mathrm{~T} 3)$ but positive allometry $(\mathrm{b}>1)$ for T2 with $\log$ values of wet weight. Positive allometry existed for log values of protein, ash, and organic content (T1, T3) but negative allometric growth was observed for $\mathrm{T} 2$ fed fish against log wet body weight (Table 4).

The values of mean condition factor for GIFT were $1.50 \pm 0.19$ with range $1.30-1.97$ for $\mathrm{T} 1$ (15\% CP), $1.43 \pm 0.14$ with range $1.22-1.63$ for T2 $(20 \% \mathrm{CP})$ and $1.29 \pm 0.28$ with range $0.75-1.73$ for T3 $(25 \%)$ diets. It did not show any effect on percent body components like protein, water, ash and organic content (T1, T2 and T3), \%fat (T1 and T3) except for \%fat (T2) which showed least positive correlation $(\mathrm{P}>0.05)$ (Table 5$)$.

The proximate composition helps in the assessment of physiological and feeding conditions of the fish (Chandrashaker et al., 2004). The results obtained on water, ash, fat, protein and organic content, expressed in grams, in the present work exhibited approximately similar values as reported by other investigators for same and other fish species (Table 1). The values of water, fat and ash content are best supported by findings of Bandarra et al. (2009), in scabbardfish, water content in Tilapia mossambis (Adefemi, 2011) ash and fat content in Nile tilapia (Jim et al., 2017), fat and protein in GIFT (De Silva et al., 2016). Our findings of protein content both wet and dry weight basis are higher than those reported by Khalid and Naeem (2018) in Ctenopharyngodon idella. These variations may be the result of different environmental conditions and diet composition (Ebrahimi and Ouraji, 2012).

The proximate analyses result of this study indicated that major component of fish body was water. The water content recorded as $79.13 \%, 80.85 \%$ and 
$78.48 \%$ for T1, T2 and T3 respectively, are similar to the findings of Adefemi (2011) 79.50\% in Tilapia mossambis and Naeem et al. (2011a) in hybrid Catla catla $\times$ Labeo rohita $(79.13 \%), 80.80 \%$ for cultured Oreochromis niloticus by Job et al. (2015) and 80.76\% for Ctenopharyngodon idella by Khalid and Naeem (2018). But our present findings deviate from the results of Osibona et al. (2009) in Clarias garipinus 74.3\% and Yousaf et al. (2011) in Wallago attu 76.19\%. These differences may be due to variations in many factors like environment, type of feed and maturity stage of fish (De Lange et al., 2003).

Table 1: Values of means and ranges of different body components of GIFT $(n=10)$.

\begin{tabular}{|c|c|c|c|}
\hline Body components & $\begin{array}{l}\text { Diet var- } \\
\text { iables }\end{array}$ & $\begin{array}{l}\text { Means } \pm \text { stand- } \\
\text { ard deviation }\end{array}$ & Ranges \\
\hline $\begin{array}{l}\text { Water contents } \\
(\%)\end{array}$ & $\begin{array}{l}\text { T1 } \\
\text { T2 } \\
\text { T3 }\end{array}$ & $\begin{array}{l}79.13 \pm 1.44 \\
80.85 \pm 1.12 \\
78.48 \pm 2.40\end{array}$ & $\begin{array}{l}76.92-81.45 \\
78.63-82.79 \\
75.14-84.09\end{array}$ \\
\hline $\begin{array}{l}\text { Ash content } \\
\text { (\%Wet weight) }\end{array}$ & $\begin{array}{l}\text { T1 } \\
\text { T2 } \\
\text { T3 }\end{array}$ & $\begin{array}{l}2.75 \pm 0.71 \\
3.27 \pm 0.47 \\
3.30 \pm 0.07\end{array}$ & $\begin{array}{l}1.34-3.75 \\
2.66-4.09 \\
1.65-4.37\end{array}$ \\
\hline $\begin{array}{l}\text { Ash content (\%dry } \\
\text { weight) }\end{array}$ & $\begin{array}{l}\text { T1 } \\
\text { T2 } \\
\text { T3 }\end{array}$ & $\begin{array}{l}13.13 \pm 3.13 \\
16.19 \pm 1.55 \\
15.47 \pm 3.50\end{array}$ & $\begin{array}{l}1.34-3.75 \\
14.09-18.15 \\
7.93-20.00\end{array}$ \\
\hline $\begin{array}{l}\text { Fat content (\%wet } \\
\text { weight) }\end{array}$ & $\begin{array}{l}\mathrm{T} 1 \\
\mathrm{~T} 2 \\
\mathrm{~T} 3\end{array}$ & $\begin{array}{l}3.74 \pm 1.65 \\
2.62 \pm 0.69 \\
2.82 \pm 1.18\end{array}$ & $\begin{array}{l}2.03-6.92 \\
1.87-3.67 \\
1.59-4.97\end{array}$ \\
\hline $\begin{array}{l}\text { Fat content (\% dry } \\
\text { weight) }\end{array}$ & $\begin{array}{l}\text { T1 } \\
\text { T2 } \\
\text { T3 }\end{array}$ & $\begin{array}{l}18.00 \pm 7.89 \\
13.37 \pm 4.19 \\
13.00 \pm 4.83\end{array}$ & $\begin{array}{l}10.00-30.00 \\
10.00-20.00 \\
10.00-20.00\end{array}$ \\
\hline $\begin{array}{l}\text { Protein contents } \\
\text { (\%wet weight) }\end{array}$ & $\begin{array}{l}\mathrm{T} 1 \\
\mathrm{~T} 2 \\
\mathrm{~T} 3\end{array}$ & $\begin{array}{l}14.43 \pm 2.00 \\
14.00 \pm 1.73 \\
15.39 \pm 1.99\end{array}$ & $\begin{array}{l}10.24-16.97 \\
10.74-16.36 \\
11.44-19.10\end{array}$ \\
\hline $\begin{array}{l}\text { Protein contents } \\
\text { (\%dry weight) }\end{array}$ & $\begin{array}{l}\text { T1 } \\
\text { T2 } \\
\text { T3 }\end{array}$ & $\begin{array}{l}69.07 \pm 8.15 \\
70.23 \pm 4.35 \\
71.53 \pm 3.84\end{array}$ & $\begin{array}{l}55.22-77.00 \\
62.38-75.00 \\
65.00-77.89\end{array}$ \\
\hline $\begin{array}{l}\text { Organic content } \\
\text { (\% wet weight) }\end{array}$ & $\begin{array}{l}\text { T1 } \\
\text { T2 } \\
\text { T3 }\end{array}$ & $\begin{array}{l}18.12 \pm 1.21 \\
16.62 \pm 1.41 \\
18.22 \pm 2.44\end{array}$ & $\begin{array}{l}15.81-19.83 \\
14.18-18.64 \\
13.03-21.55\end{array}$ \\
\hline $\begin{array}{l}\text { Organic content } \\
\text { (\% dry weight) }\end{array}$ & $\begin{array}{l}\text { T1 } \\
\text { T2 } \\
\text { T3 }\end{array}$ & $\begin{array}{l}82.06 \pm 17.09 \\
83.60 \pm 1.49 \\
84.53 \pm 3.50\end{array}$ & $\begin{array}{c}82.06-93.04 \\
81.85-85.91 \\
80.00-92.07\end{array}$ \\
\hline
\end{tabular}

The results of the present study represented lowest water contents and highest protein content in GIFT fed with $25 \%$ dietary protein. These findings coincide with those reported by Bano et al. (2019) in Labeo calbasu and Ishtiaq and Naeem (2019) in Catla catla who had studied the effect of dietary protein levels on body composition. They found highest protein and lowest water content in fish samples fed upon $25 \% \mathrm{CP}$.
In Labeo robita fingerlings, increase in lipid and protein with decrease in water content had been reported with dietary protein levels (Satpathy et al., 2003).

The relationship between percent water and proximate components (fat, ash, protein and organic content) is used as predictors of fish body composition. In the present study, equations were developed between percent water and percent of each of proximate constituents which showed non-significant relation $(P>0.05)$ except for percent fat dry weight (T2,), \% organic wet weight (T1, T2, T3) and \% protein wet weight (T3). The results of relationships of $\%$ water and each percent composition parameters are best supported by Naeem et al. (2011a) in hybrid Catla catla $\times$ Labeo rohita and Bano et al. (2019) in L. calbasu.

Previously many studies indicated inverse relationship between percent water, percent fat and percent protein in fish whole body (Yousaf et al., 2011; Naeem et al., 2011b). The present work examined that relation of water and fat wet weight was not significant as shown by predictive equations. Although fat dry weight was found significant in T2 fed GIFT while non-significant inverse relationship was observed between percent water and percent fat in $\mathrm{T} 1$ and $\mathrm{T} 3$ (wet weight) and T3 (fat dry weight). Naeem et al. (2013) and Khalid and Naeem (2018) have reported the same trend.

Percent water showed strong inverse correlation with percent protein wet weight in T3 and strongly inverse correlation with organic content in $\mathrm{T} 1$ and T3 while direct least significant in T2 diet fed GIFT. Similar results have been documented by Naeem et al. (2011a) in hybrid (Catla catla $\times$ labeo rohita). Naeem and Ishtiaq (2011) also documented strong inverse correlation between percent water and organic content. The present findings declared non-significant relationships of $\%$ water and ash (wet and dry weight) in T1, T2 and T3. Naeem et al. (2011a) reported similar non-significant relation in hybrid Catla catla $\times$ Labeo robita.

Predictive equations were made to observe the influence of body size on the total body constituents of studied GIFT. The results showed definite influence of wet weight and total length on various body constituents of GIFT fed upon different dietary protein. The relationship between $\log$ transformed data of wet body weight as well as total length versus 
Table 2: Relation between percent water and percent components of GIFT body $(n=10)$.

\begin{tabular}{|c|c|c|c|c|c|c|c|}
\hline Relations & $\begin{array}{l}\text { Diet varia- } \\
\text { bles }\end{array}$ & $\begin{array}{l}\text { Correlation coeffi- } \\
\text { cients }(r)\end{array}$ & $\begin{array}{l}\text { intercept } \\
\text { (a) }\end{array}$ & $\begin{array}{l}\text { slope } \\
\text { (b) }\end{array}$ & $\begin{array}{l}\text { Standard error } \\
\text { of }(\mathbf{b})\end{array}$ & $\begin{array}{l}t \text { value when } \\
b=0\end{array}$ & p-value \\
\hline $\begin{array}{l}\text { \% Water (x) } \\
\text { \%Fat wet weight (y) }\end{array}$ & $\begin{array}{l}\text { T1 } \\
\text { T2 } \\
\text { T3 }\end{array}$ & $\begin{array}{l}0.010^{\text {ns }} \\
0.286^{\text {ns }} \\
0.472^{\text {ns }}\end{array}$ & $\begin{array}{l}4.65 \\
-11.74 \\
20.35\end{array}$ & $\begin{array}{l}-0.0116 \\
0.1777 \\
-0.2233\end{array}$ & $\begin{array}{l}0.403 \\
0.211 \\
0.147\end{array}$ & $\begin{array}{l}-0.029 \\
0.844 \\
-1.515\end{array}$ & $\begin{array}{l}0.978^{\text {ns }} \\
0.423^{\text {ns }} \\
0.168^{\text {ns }}\end{array}$ \\
\hline $\begin{array}{l}\text { \% Water (x) } \\
\text { \%Fat dry weight (y) }\end{array}$ & $\begin{array}{l}\text { T1 } \\
\text { T2 } \\
\text { T3 }\end{array}$ & $\begin{array}{l}0.193^{\text {ns }} \\
0.787^{\text {** }} \\
0.247^{\text {ns }}\end{array}$ & $\begin{array}{l}-65.55 \\
8.24 \\
50.47\end{array}$ & $\begin{array}{l}1.056 \\
2.4967 \\
-0.4775\end{array}$ & $\begin{array}{l}1.894 \\
0.692 \\
0.661\end{array}$ & $\begin{array}{l}0.557 \\
3.609 \\
-0.722\end{array}$ & $\begin{array}{l}0.592^{\text {ns }} \\
0.007^{\text {** }} \\
0.491^{\text {ns }}\end{array}$ \\
\hline $\begin{array}{l}\text { \% Water }(\mathrm{x}) \\
\text { \% Protein wet weight }(\mathrm{y})\end{array}$ & $\begin{array}{l}\text { T1 } \\
\text { T2 } \\
\text { T3 }\end{array}$ & $\begin{array}{l}0.521^{\mathrm{ns}} \\
0.624^{\mathrm{ns}} \\
0.893^{* * *}\end{array}$ & $\begin{array}{l}71.52 \\
6.46 \\
71.17\end{array}$ & $\begin{array}{l}-0.7215 \\
2.3048 \\
-0.7108\end{array}$ & $\begin{array}{l}0.417 \\
1.021 \\
0.126\end{array}$ & $\begin{array}{l}-1.729 \\
2.257 \\
-5.624\end{array}$ & $\begin{array}{l}0.122^{\text {ns }} \\
0.054^{\text {ns }} \\
0.000^{\text {**** }}\end{array}$ \\
\hline $\begin{array}{l}\text { \% Water (x) } \\
\text { \% Protein dry weight }(\mathrm{y})\end{array}$ & $\begin{array}{l}\text { T1 } \\
\text { T2 } \\
\text { T3 }\end{array}$ & $\begin{array}{l}0.071^{\text {ns }} \\
0.101^{\text {ss }} \\
0.019^{\text {ns }}\end{array}$ & $\begin{array}{l}100.86 \\
67.16 \\
69.21\end{array}$ & $\begin{array}{l}-0.4018 \\
0.9377 \\
0.0296\end{array}$ & $\begin{array}{l}1.991 \\
3.258 \\
0.542\end{array}$ & $\begin{array}{l}-0.202 \\
0.288 \\
0.055\end{array}$ & $\begin{array}{l}0.845^{\text {ns }} \\
0.781^{\text {ns }} \\
0.957^{\text {ns }}\end{array}$ \\
\hline $\begin{array}{l}\text { \%Water }(\mathrm{x}) \\
\text { \%Ash wet weight (y) }\end{array}$ & $\begin{array}{l}\text { T1 } \\
\text { T2 } \\
\text { T3 }\end{array}$ & $\begin{array}{l}0.541^{\text {ns }} \\
0.204^{\text {ns }} \\
0.226^{\text {ns }}\end{array}$ & $\begin{array}{l}23.79 \\
3.61 \\
8.48\end{array}$ & $\begin{array}{l}-0.6498 \\
-0.3011 \\
-0.0659\end{array}$ & $\begin{array}{l}0.730 \\
0.511 \\
0.100\end{array}$ & $\begin{array}{l}-0.889 \\
-0.589 \\
-0.657\end{array}$ & $\begin{array}{l}0.106^{\mathrm{ns}} \\
0.572^{\text {ns }} \\
0.529^{\text {ns }}\end{array}$ \\
\hline $\begin{array}{l}\text { \% Water (x) } \\
\text { \%Ash dry weight (y) }\end{array}$ & $\begin{array}{l}\text { T1 } \\
\text { T2 } \\
\text { T3 }\end{array}$ & $\begin{array}{l}0.300^{\text {ns }} \\
0.134^{\text {ns }} \\
0.320^{\text {ns }}\end{array}$ & $\begin{array}{l}64.55 \\
30.89 \\
-19.68\end{array}$ & $\begin{array}{l}-0.6498 \\
-0.1792 \\
0.4479\end{array}$ & $\begin{array}{l}0.729 \\
0.468 \\
0.469\end{array}$ & $\begin{array}{l}-0.891 \\
-0.383 \\
0.956\end{array}$ & $\begin{array}{l}0.399^{\text {ns }} \\
0.711^{\text {ns }} \\
0.367^{\text {ns }}\end{array}$ \\
\hline $\begin{array}{l}\% \text { Water }(\mathrm{x}) \\
\% \text { Organic contents wet } \\
\text { weight }(\mathrm{y})\end{array}$ & $\begin{array}{l}\text { T1 } \\
\text { T2 } \\
\text { T3 }\end{array}$ & $\begin{array}{l}0.874^{* * * *} \\
0.668^{*} \\
0.957^{* * *}\end{array}$ & $\begin{array}{l}76.20 \\
10.07 \\
91.52\end{array}$ & $\begin{array}{l}-0.7340 \\
2.0037 \\
-0.9340\end{array}$ & $\begin{array}{l}0.144 \\
0.789 \\
0.100\end{array}$ & $\begin{array}{l}-5.083 \\
2.54 \\
-9.309\end{array}$ & $\begin{array}{l}0.000^{\text {**** }} \\
0.035^{*} \\
0.000^{\text {*** }}\end{array}$ \\
\hline $\begin{array}{l}\% \text { Water }(\mathrm{x}) \\
\% \text { Organic contents dry } \\
\text { weight }(\mathrm{y})\end{array}$ & $\begin{array}{l}\text { T1 } \\
\text { T2 } \\
\text { T3 }\end{array}$ & $\begin{array}{l}0.308^{\text {ns }} \\
0.134^{\text {ns }} \\
0.320^{\text {ns }}\end{array}$ & $\begin{array}{l}35.46 \\
69.11 \\
119.68\end{array}$ & $\begin{array}{l}0.6498 \\
0.1792 \\
-0.4479\end{array}$ & $\begin{array}{l}0.730 \\
0.468 \\
0.469\end{array}$ & $\begin{array}{l}0.889 \\
0.383 \\
-0.956\end{array}$ & $\begin{array}{l}0.399^{\text {ns }} \\
0.712^{\text {ns }} \\
0.367^{\text {ns }}\end{array}$ \\
\hline
\end{tabular}

${ }^{* * 0 * k *} P<0.001 ; * * * 0.01 ; * P<0.05 ; n s P>0.05$

Table 3: Relation between log wet weight $(g)$ and total log components of GIFT body $(n=10)$.

\begin{tabular}{|c|c|c|c|c|c|c|c|}
\hline Relations & $\begin{array}{l}\text { Diet Varia- } \\
\text { bles }\end{array}$ & $\begin{array}{l}\text { Correlation coeffi- } \\
\text { cients (r) }\end{array}$ & $\begin{array}{l}\text { intercept } \\
\text { (a) }\end{array}$ & $\begin{array}{l}\text { Slope } \\
\text { (b) }\end{array}$ & $\begin{array}{l}\text { Standard } \\
\text { error of }(b)\end{array}$ & $\begin{array}{l}\text { Value of } t \\
\text { when } b=1\end{array}$ & P-value \\
\hline $\begin{array}{l}\log \text { body weight }(\mathrm{x}) \\
\log \text { water content }(\mathrm{y})\end{array}$ & $\begin{array}{l}\text { T1 } \\
\text { T2 } \\
\text { T3 }\end{array}$ & $\begin{array}{l}0.997^{\text {**** }} \\
0.987^{* * * *} \\
0.980^{* * * *}\end{array}$ & $\begin{array}{l}-0.08 \\
-0.16 \\
0.02\end{array}$ & $\begin{array}{l}0.9771 \\
1.0639 \\
0.8968\end{array}$ & $\begin{array}{l}0.029 \\
0.061 \\
0.064\end{array}$ & $\begin{array}{l}33.854 \\
17.363 \\
14.088\end{array}$ & $\begin{array}{l}0.000^{* * *} \\
0.000^{* * *} \\
0.000^{* * *}\end{array}$ \\
\hline $\begin{array}{l}\text { log body weight, } \mathrm{g}(\mathrm{x}) \\
\log \text { fat content }(\mathrm{y})\end{array}$ & $\begin{array}{l}\text { T1 } \\
\text { T2 } \\
\text { T3 }\end{array}$ & $\begin{array}{l}0.387^{\text {ns }} \\
0.088^{\text {ns }} \\
0.415^{\text {ns }}\end{array}$ & $\begin{array}{l}-1.31 \\
-0.35 \\
-1.76\end{array}$ & $\begin{array}{l}0.8628 \\
-0.1633 \\
1.1537\end{array}$ & $\begin{array}{l}0.727 \\
0.657 \\
0.894\end{array}$ & $\begin{array}{l}1.187 \\
-0.248 \\
1.290\end{array}$ & $\begin{array}{l}0.629^{\text {ns }} \\
0.081^{\text {ns }} \\
0.233^{\text {ns }}\end{array}$ \\
\hline $\begin{array}{l}\log \text { body weight }(\mathrm{x}) \\
\log \text { protein content }(\mathrm{y})\end{array}$ & $\begin{array}{l}\text { T1 } \\
\text { T2 } \\
\text { T3 }\end{array}$ & $\begin{array}{l}0.833^{* *} \\
0.591^{\text {ns }} \\
0.874^{* *}\end{array}$ & $\begin{array}{l}-0.99 \\
-0.631 \\
-1.29\end{array}$ & $\begin{array}{l}1.1190 \\
0.7885 \\
1.4056\end{array}$ & $\begin{array}{l}0.263 \\
0.381 \\
0.276\end{array}$ & $\begin{array}{l}4.259 \\
2.071 \\
0.708\end{array}$ & $\begin{array}{l}0.002^{* *} \\
0.072^{\text {ns }} \\
0.000^{* * * *}\end{array}$ \\
\hline $\begin{array}{l}\log \text { body weight }(\mathrm{x}) \\
\log \text { ash content }(\mathrm{y})\end{array}$ & $\begin{array}{l}\text { T1 } \\
\text { T2 } \\
\text { T3 }\end{array}$ & $\begin{array}{c}0.709^{*} \\
0.584^{\text {ns }} \\
0.708^{*}\end{array}$ & $\begin{array}{l}-1.97 \\
-1.34 \\
-2.23\end{array}$ & $\begin{array}{l}1.3354 \\
0.8621 \\
1.6284\end{array}$ & $\begin{array}{l}0.470 \\
0.424 \\
0.574\end{array}$ & $\begin{array}{l}2.841 \\
2.034 \\
0.574\end{array}$ & $\begin{array}{l}0.021^{*} \\
0.076^{\text {ns }} \\
0.021^{*}\end{array}$ \\
\hline $\begin{array}{l}\log \text { body weight }(x) \\
\log \text { organic contents }(y)\end{array}$ & $\begin{array}{l}\text { T1 } \\
\text { T2 } \\
\text { T3 }\end{array}$ & $\begin{array}{l}0.962^{* * * *} \\
0.717^{*} \\
0.844^{* *}\end{array}$ & $\begin{array}{l}-0.82 \\
-0.41 \\
-1.17\end{array}$ & $\begin{array}{l}1.0693 \\
0.6524 \\
1.3636\end{array}$ & $\begin{array}{l}0.107 \\
0.224 \\
0.306\end{array}$ & $\begin{array}{l}10.023 \\
2.909 \\
4.449\end{array}$ & $\begin{array}{l}0.000^{* * *} \\
0.019^{*} \\
0.002^{* *}\end{array}$ \\
\hline
\end{tabular}

$P>0.05=n s ; * * * * 0.001 ; * * * 0.01 ;{ }^{*} P<0.05$

total water content (Tables 3 and 4) exhibited strong correlation $(P<0.001)$ for T1, T2 and T3. These findings are supported by Naeem and Salam (2010) in bighead carp, Naeem et al. (2011a) in Catla catla $\times$ Labeo robita hybrid, Naeem and Ishtiaq (2011) in Mystus bleekeri,
Naeem et al. (2016) in rainbow trout, Khalid and Naeem (2018) in Ctenopharyngodon idella and Bano et al. (2019) in L. calbasu reported similar results.

Log transformed data showed non-significant correlation 
Table 4: Relation between log total length $(\mathrm{cm})$ and log total components of GIFT body $(n=10)$.

\begin{tabular}{|c|c|c|c|c|c|c|c|}
\hline Relations & Diet variables & $\begin{array}{l}\text { Correlation } \\
\text { coefficients (r) }\end{array}$ & intercept (a) & Slope (b) & $\begin{array}{l}\text { Standard } \\
\text { error of } b\end{array}$ & $\begin{array}{l}\text { t value when } \\
b=3\end{array}$ & p-value \\
\hline log total length (x) & $\mathrm{T} 1$ & $0.936^{* * *}$ & -1.12 & 2.1711 & 0.288 & 7.54 & $0.000^{* * *}$ \\
\hline \multirow[t]{2}{*}{$\log$ water content $(y)$} & $\mathrm{T} 2$ & $0.943^{* * * *}$ & -0.7184 & 1.7417 & 0.2169 & 8.0277 & $0.000^{* * * *}$ \\
\hline & T3 & $0.965^{\text {**** }}$ & 0.00354 & 1.0427 & 0.099 & 10.531 & $0.000^{* * * *}$ \\
\hline log total length $(x)$ & $\mathrm{T} 1$ & $0.216^{\mathrm{ns}}$ & -1.45 & 1.1406 & 0.288 & 7.544 & $0.548^{\mathrm{ns}}$ \\
\hline \multirow{2}{*}{$\log$ fat content $(y)$} & $\mathrm{T} 2$ & $0.247^{\mathrm{ns}}$ & 0.24 & -0.7896 & 1.095 & 1.095 & $0.491^{\mathrm{ns}}$ \\
\hline & T3 & $0.265^{\mathrm{ns}}$ & -1.29 & 0.8699 & 1.119 & 0.778 & $0.459^{\mathrm{ns}}$ \\
\hline log total length (x) & $\mathrm{T} 1$ & $0.745^{*}$ & -2.05 & 2.3674 & 0.749 & 3.161 & $0.013^{*}$ \\
\hline \multirow[t]{2}{*}{ log protein content $(y)$} & $\mathrm{T} 2$ & $0.671^{*}$ & -1.28 & 1.5335 & 0.599 & 2.558 & $0.030^{*}$ \\
\hline & $\mathrm{T} 3$ & $0.808^{*}$ & -1.21 & 1.5342 & 0.395 & 3.884 & $0.004^{* *}$ \\
\hline log total length $(\mathrm{x})$ & $\mathrm{T} 1$ & $0.605^{\mathrm{ns}}$ & -3.10 & 2.6944 & 1.255 & 2.147 & $0.064^{\mathrm{ns}}$ \\
\hline \multirow[t]{2}{*}{ log ash content (y) } & $\mathrm{T} 2$ & $0.657^{*}$ & -2.03 & 1.6617 & 0.675 & 2.463 & $0.030^{*}$ \\
\hline & T3 & $0.679^{*}$ & -2.20 & 1.8425 & 0.705 & 2.613 & $0.039^{*}$ \\
\hline log total length $(x)$ & $\mathrm{T} 1$ & $0.815^{\text {** }}$ & -1.72 & 2.1410 & 0.538 & 3.976 & $0.004^{* *}$ \\
\hline \multirow[t]{2}{*}{ log organic contents $(y)$} & $\mathrm{T} 2$ & $0.755^{*}$ & -0.86 & 1.1777 & 0.361 & 3.262 & $0.011^{*}$ \\
\hline & T3 & $0.744^{*}$ & -1.02 & 1.4190 & 0.451 & 3.149 & $0.014^{*}$ \\
\hline
\end{tabular}

***** $P<0.001 ;{ }^{* *} P<0.01 ;{ }^{*} P<0.05, P>0.05=n s$

Table 5: Relation between condition factor and percent components of GIFT body $(n=10)$.

\begin{tabular}{|c|c|c|c|c|c|c|c|}
\hline Relations & $\begin{array}{l}\text { Diet varia- } \\
\text { bles }\end{array}$ & $\begin{array}{l}\text { Correlation coeffi- } \\
\text { cients }(\mathbf{r})\end{array}$ & $\begin{array}{l}\text { intercept } \\
\text { (a) }\end{array}$ & Slope (b) & $\begin{array}{l}\text { Standard } \\
\text { error of } b\end{array}$ & $\begin{array}{l}\text { Value of } t \\
\text { when } b=0\end{array}$ & p-value \\
\hline $\begin{array}{l}\text { condition factor }(\mathrm{x}) \\
\% \text { water }(\mathrm{y})\end{array}$ & $\begin{array}{l}\text { T1 } \\
\text { T2 } \\
\text { T3 }\end{array}$ & $\begin{array}{l}0.509^{\text {ns }} \\
0.102^{\text {ns }} \\
0.304^{\text {ns }}\end{array}$ & $\begin{array}{l}85.06 \\
82.04 \\
75.01\end{array}$ & $\begin{array}{l}-3.9706 \\
-0.8309 \\
2.6806\end{array}$ & $\begin{array}{c}2.369 \\
2.875 \\
2.974\end{array}$ & $\begin{array}{l}-1.676 \\
-0.289 \\
0.901\end{array}$ & $\begin{array}{l}0.132^{\text {ns }} \\
0.779^{\text {s }} \\
0.394^{\text {ns }}\end{array}$ \\
\hline $\begin{array}{l}\text { Condition factor }(\mathrm{x}) \\
\% \text { Fat }(\mathrm{y})\end{array}$ & $\begin{array}{l}\text { T1 } \\
\text { T2 } \\
\text { T3 }\end{array}$ & $\begin{array}{c}0.538^{\mathrm{ns}} \\
0.728^{*} \\
0.201^{\mathrm{ns}}\end{array}$ & $\begin{array}{l}-3.41 \\
-2.68 \\
1.74\end{array}$ & $\begin{array}{l}4.779 \\
3.6995 \\
0.8390\end{array}$ & $\begin{array}{c}2.649 \\
1.231 \\
1.446\end{array}$ & $\begin{array}{l}1.804 \\
3.005 \\
0.580\end{array}$ & $\begin{array}{l}0.109^{\text {ns }} \\
0.017^{*} \\
0.578^{\text {ns }}\end{array}$ \\
\hline $\begin{array}{l}\text { Condition factor }(\mathrm{x}) \\
\% \text { Protein }(\mathrm{y})\end{array}$ & $\begin{array}{l}\text { T1 } \\
\text { T2 } \\
\text { T3 }\end{array}$ & $\begin{array}{l}0.123^{\text {ns }} \\
0.100^{\text {ns }} \\
0.364^{\text {ns }}\end{array}$ & $\begin{array}{l}16.41 \\
15.83 \\
18.69\end{array}$ & $\begin{array}{l}-1.3282 \\
-1.2745 \\
-2.5559\end{array}$ & $\begin{array}{c}3.781 \\
4.469 \\
2.313\end{array}$ & $\begin{array}{l}-0.351 \\
-0.285 \\
-1.105\end{array}$ & $\begin{array}{l}0.734^{\text {ns }} \\
0.783^{\text {ns }} \\
0.301^{\text {ns }}\end{array}$ \\
\hline $\begin{array}{l}\text { Condition factor }(\mathrm{x}) \\
\% \text { Ash }(\mathrm{y})\end{array}$ & $\begin{array}{l}\text { T1 } \\
\text { T2 } \\
\text { T3 }\end{array}$ & $\begin{array}{l}0.058^{\text {ns }} \\
0.148^{\text {ns }} \\
0.375^{\text {ns }}\end{array}$ & $\begin{array}{l}2.42 \\
3.99 \\
4.55\end{array}$ & $\begin{array}{l}0.2216 \\
-0.509 \\
-0.9637\end{array}$ & $\begin{array}{c}1.351 \\
1.202 \\
0.843\end{array}$ & $\begin{array}{l}0.164 \\
-0.684 \\
-1.143\end{array}$ & $\begin{array}{l}0.874^{\text {ns }} \\
0.683^{\text {ns }} \\
0.286^{\text {ns }}\end{array}$ \\
\hline $\begin{array}{l}\text { Condition factor }(\mathrm{x}) \\
\% \text { Organic contents }(\mathrm{y})\end{array}$ & $\begin{array}{l}\text { T1 } \\
\text { T2 } \\
\text { T3 }\end{array}$ & $\begin{array}{l}0.569^{\text {ns }} \\
0.235^{\text {ns }} \\
0.199^{\text {ns }}\end{array}$ & $\begin{array}{l}12.55 \\
13.14 \\
20.44\end{array}$ & $\begin{array}{l}3.7255 \\
2.4249 \\
-1.7169\end{array}$ & $\begin{array}{c}1.902 \\
3.545 \\
2.986\end{array}$ & $\begin{array}{l}1.959 \\
0.684 \\
-0.575\end{array}$ & $\begin{array}{l}0.086^{\text {ns }} \\
0.153^{\text {ns }} \\
0.581^{\text {ns }}\end{array}$ \\
\hline
\end{tabular}

$P>0.05=n s ;{ }^{*} P<0.05$

$(P>0.05)$ with total fat content versus body weight and total length for T1, T2 and T3 which is contrary to Naeem and Ishtiaq (2011), Naeem et al. (2011a) and Naeem and Salam, 2010. Khalid and Naeem (2018) also documented strong correlation between log values of total length and body weight with all the body constituents in Ctenopharyngodon Idella. The reasons of such variations may be the result of differences in the geography, age, season and sampling type (Abbasi et al., 2017), variations in nutrition as well as size of fish body (Ebrahimi and Quraji, 2012).

The values of slope ' $b$ ' shows isometric state when $b=1$ on $\log$ transformed relationships. The value of $\mathrm{b}>1$ in log water (T2), log fat (T3), log protein, log ash and $\log$ organic content $(\mathrm{T} 1, \mathrm{~T} 3)$ indicated positive allometry i.e. these body constituents increased with increasing body weight. Although, negative allometry was observed in $\log$ water $(\mathrm{T} 1, \mathrm{~T} 3), \log$ fat $(\mathrm{T} 1, \mathrm{~T} 2)$, log protein, log ash and log organic content (T2). The negative allometry in log water is supported by findings of Naeem and Ishtiaq (2011), Naeem et al. (2011a, b, c), Naeem et al. (2016), Naeem et al. (2011a, c) and Naeem and Ishtiaq (2011) for log ash content and Naeem et al. (2011a, b) for log fat. Naeem and Ishtiaq (2011) reported positive allometry for $\log$ fat, 
$\log$ protein and log organic content which support our findings in $\log$ fat (T3), log protein, ash and organic content $(\mathrm{T} 1, \mathrm{~T} 3)$. Positive allometry in $\log$ fat is also supported by Naeem et al. (2016) in rainbow trout. Negative allometry in water $(\mathrm{T} 1, \mathrm{~T} 3)$ and fat (T1, T2) coincides with findings of Naeem et al. (2011 a, b), while log water (T1,T3) and log ash (T2) are supported by Naeem et al. (2011c), Naeem and Ishtiaq (2011) and Naeem et al. (2016). Although, all the body components exhibited negative allometry $(b<3)$ with total length showing deviation from cube law.

Most of the regression relationships between condition factor and various body constituents showed nonsignificant correlation except for $\%$ fat suggesting no influence of condition factor on these constituents. Khalid and Naeem (2018) has also documented non-significant correlation between condition factor and \% fat, \%protein, \%ash while least significant correlation with water and organic content. Naeem et al. (2011b), Naeem and Ishtiaq (2011) and Naeem et al. (2017) have also reported similar non-significant findings in all the body constituents of female farmed Oreochromis mossambicus and Mystus bleekeri and Cirrbinus mrigala, respectively.

\section{Conclusions and Recommendations}

The present study concludes that different levels of dietary proteins and body size have definite influence on the proximate composition. It also assists to model growth of fish and protein deposition rate at graded dietary protein levels. The findings of the investigation confirmed that proximate composition parameters show variation with different protein diets. The obtained results reveal that GIFT possesses more amount of protein and low fat contents fed upon 25\% $\mathrm{CP}$ diet. It will contribute to the nutritional qualities and growth of human beings as indicated by high protein content and is best for human consumption.

\section{Novelty Statement}

Results of this study shows that highest protein contents of Genetically Improved Farmed Tilapia (GIFT) can be achieved by feeding a diet containing $25 \%$ crude protein.

\section{Author's Contribution}

Anila Kousar conducted the experiment and lab work, collected and analyzed data and wrote the manuscript. Muhammad Naeem supervised the research work, made available the necessary circumstances for the completion of experiment and assisted in the manuscript writing. Samrah Masud helped in data analysis.

\section{Conflict of interest}

The authors declare that they have no conflict of interest.

\section{References}

Adefemi, O.S., 2011. Chemical composition of Tilapia mosambis fish from major dams in EkitiState, Nigeria. Afr. J. F. Sci., 5(10): 550-554.

Abbasi, G.H., A. Kamali, M. Soltani and M. Sharifian, 2017. Effects of zingiber officinale powder on growth parameters, survival rate and biochemical composition of body in juvenile common carp (Cyprinus carpio). Iran. J. Fish. Sci., 16(1): 67-85.

Azam, K., M.Y. Ali, M. Asaduzzaman, M.Z. Basher and M.M. Hossain. 2004. Biochemical assessment of selected fresh fish. J. Biol. Sci., 4: 9-10.

Bandarra, N.M., I. Batista and M.L. Maria LeoNor Nunes, 2009. Chemical composition and nutritional value of raw and cooked black scabbardfish (Aphanopus carbo). Sci. Mar. 73(2): 105-113. https://doi.org/10.3989/ scimar.2009.73s2105

Bano, S., M. Naeem and S. Masud. 2019. Effect of different dietary protein levels on proximate composition of Labeo calbasu from Pakistan. Int. J. Biol. Pham. Allied Sci. 8(11): 2095-2130. https://doi.org/10.31032/ IJBPAS/2019/8.11.4855

Bennett, A., P. Patil, K. Kleisner, D. Rader, J. Virdin and X. Basurto. 2018. Contribution of fisheries to food and nutrition security: Current knowledge, policy, and research. NI Report 1802. Durham, NC: Duke University.

Bentsen, H.B., A.E. Eknath, M.S.P. Vera, J.C., Danting,H.L.Bolivar,R.A.Reyes,E.E.Dionisio, F.M. Longalong, A.V. Circa, M.M. Tayamen and B. Gjerd. 1998. Genetic improvement of farmed tilapias: growth performance in a complete diallel cross experiment with eight strains of Oreochromis niloticus. Aquacult. Amsterdam., 160(1-2): 145- 173. https://doi. 
org/10.1016/S0044-8486(97)00230-5

Bligh, E.G. and W.J. Dyer, 1959. A rapid method of total lipid extraction and purification. Can. J. Biochem. Physiol., 37: 911-917. https://doi. org/10.1139/059-099

Chandrashekhar, A., P. Rao and A.B. Abidi. 2004. Changes in muscle biochemical composition of Labeo rohita (Ham.) in relation to season. India. J. Fish. 51: 319-323.

Caulton, M.S. and E. Bursell, 1977. The relationship between changes in condition and body composition in young Tilapia rendalli. J. Fish Biol., 11: 1443-1450. https://doi. org/10.1111/j.1095-8649.1977.tb04107.x

De Lange, C.F.M., P.C.H. Morel and S. H. Brikett. 2003. Modeling chemical and physical body composition of the growing pig. Am. Soc. Anim. Sci., 81(2): 159-165.

Degani, G., 1988. Body composition of African catfish (Clarias gariepinus) at different ages. Israeli J. Aquacult. Bamidgeh., 40: 118-121.

De Silva, M.P.K.S.K., W. Senaarachchi and N.P.P. Liyanag. 2016. Title-Combinatory effects of diets with three protein levels and two fat levels on growth performance and fillet composition of cage cultured genetically improved farmed tilapia (GIFT). J. Aquact. Res. Dev., S2: 008. https://doi.org/10.4172/2155-9546.S2-008

Dumas, A.,J. France and D. Bureau.2010. Modeling of growth and body composition in fish nutrition: where have we been and where are we going? Aquacult. Res., 41: 161-181. https://doi. org/10.1111/j.1365-2109.2009.02323.x

Ebrahimi, I.G. and H. Ouraji. 2012. Growth performance and body composition of kutum fingerlings, Rutilus Frisii kutum (Kamenskii, 1901 ), in response to dietary protein levels. Turk. J. Zool., 36: 551-558.

Eknath, A.E. and B.O. Acosta. 1998. Genetic improvement of farmed tilapias (GIFT) project: Final report, March 1988 to December 1997. Int. Center Living Aquat. Resour. Manage., Makati City, Philippines.

Fawole, O.O., M.A. Ogundiran, T.A. Ayandiran, and O.F. Olagunju. 2007. Proximate and Mineral Composition in Some Selected Fresh Water Fishes in Nigeria, Int. J. Food Saf., 9: 5255.

Foran, J.A., D.O. Carpenter, M.C. Hamilton, B.A. Knuth and S.J. Schwager. 2005. Riskbased consumption advice for farmed Atlantic and wild pacific salmon contaminated with dioxins and dioxin like compounds. Environ. Health Perspect.,33:552-556. https://doi.org/10.1289/ ehp.7626

Ishtiaq, A. and M. Naeem. 2019. Effect of dietary protein levels on body composition of Catla catla from Pakistan. Sindh Univ. Res. J. (Sci. Ser.) 51(2): 309-318. https://doi.org/10.26692/ sujo/2019.6.51

James, D., 1998. Production, consumption and demand.In: Fish drying and smoking production and quality. Ed. (Peter E. Doe). Technomic Publishing Company. Inc., U.S.A. pp. 1-12. https://doi.org/10.1201/9780203756003-1

Jim, F., P. Garamumhango and C. Musara. 2017. Comparative Analysis of Nutritional Value of Oreochromis niloticus (Linnaeus), Nile Tilapia, meat from three different ecosystems. J. Food Qual. Volume 2017. 1-8. Article ID 6714347. https://doi.org/10.1155/2017/6714347

Job, B.E., E.E. Antai, A.P. Inyang-Etoh, G.A. Oto-go and H.S. Ezekiel. 2015. Proximate composition and mineral contents of cultured and wild tilapia (Oreochromis niloticus), (Pisces: Cichlidae), (Linnaeus, 1758). Pak. J. Nut., 14: 195. https://doi.org/10.3923/pjn.2015.195.200

Khalid, M. and M. Naeem. 2018. Proximate analysis of grass carp (Ctenopharyngodon idella) from Southern Punjab, Pakistan. Sarhad J. Agric., 34(3): 632-639. https://doi.org/10.17582/ journal.sja/2018/34.3.632.639

Lie, O., 2001. Flesh quality the role of nutrition. Aquacult. Res., 32(1): 341-348. https://doi. org/10.1046/j.1355-557x.2001.00026.x

Naeem, M. and A. Ishtiaq. 2011. Proximate composition of Mystus bleekeri in relation to body size and condition factor from Nala Dhaik, Sialkot, Pakistan, Afr. J. Biotechnol., 10(52): 10765-10773. https://doi.org/10.5897/ AJB10.2339

Naeem, M., I. Ishtiaq and S. Naz. 2017. Influence of dietary protein on proximate composition of mori, Cirrbinus mrigala (Hamilton). MSP., 1(2): 01-05. https://doi.org/10.26480/ msp.02.2017.01.05

Naeem, M., A. Rasul, A. Salam, M.S. Iqbal, A. Ishtiaq, M. Khalid and M. Athar. 2011c. Proximate analysis of female population of wild feather back fish (Notopterus notopterus) in relation to body size and condition factor. Afr. J. Biotechnol., 10(19): 3867-3871. 
Naeem, M. and A. Salam. 2010. Proximate composition of fresh water Bighead carp, Aristichthys nobilis in relation to body size and condition factor from, Islamabad. Afr. J. Biotechnol., 9(50): 8687- 8692.

Naeem, M., A. Salam, N.T. Narejo and A. Tahir. 2013. Body composition of edible portion of wild bighead carp Aristichthys nobilis in relation to body size and condition factor from Indus River, Pakistan. Sindh Univ. Res. J. Surj., (Sci. Series). 45: 353-356.

Naeem, M., A. Salam, M. A. Bashir, A. Ishtiaq, Q. Gillani and A. Salam. 2011a. Effect of Body Size and Condition Factor on Whole Body Composition of Hybrid (Catla catla $\widehat{\partial} \times$ Labeo robita +) from Pakistan. Int. J. Bioeng. Life Sci., 5(11): 823-826.

Naeem, M., A. Salam, A. Ishtiaq, R. Baby and S.A. Rasool. 2011b. Study of Body Composition of Female Population of Farmed Oreochromis mossambicus in relation to Body Size and Condition Factor from Pakistan. Int. Conf. Biosci., Biochem. Bioinform. (IPCBEE 2011) 5: IACSIT Press, Singapore. https://doi. org/10.1109/ICCCENG.2010.5560382

Naeem, M., A. Salam, N.T. Narejo, A.A. Tahir. 2013. Body composition of edible portion of wild Bighead carp Aristichthys nobilis in relation to body size and condition factor from Indus River, Pakistan. Sindh Univ. Res. J., (Sci. Ser.) 45(2): 353-356.

Naeem, M., A. Salam and A. Zuberi. 2016. Proximate composition of freshwater rainbow trout (Oncorbynchus mykiss) in relation to body size and condition factor from Pakistan. Pak. J. Agric. Sci., 53(2): 497-502. https://doi. org/10.21162/PAKJAS/16.2653

Nettleton, J.A., 1992. Seafood nutrition in the 1990's: Issues for the consumer. In: Bligh EG. Ed Seafood Science and Technology. London: Fishing New Books. pp. 34-35.

NRC. 1993. Nutrient requirements of fish. National research council. Committee on animal nutrition.

Osibona, A.O., K.1. Kusemiju and G.R. Akande. 2009. Fatty acid composition and amino acid profile of two freshwater species, African catfish (Clarias gariepinus) and tilapia (Tilapia zillii). Afr. J. Food Agric. Nutr. Dev., 9(1): 608-621. https://doi.org/10.4314/ajfand.v9i1.19216

Salam, A. and P.M.C. Davies. 1994. Body composition of northern pike, Esox lucius $\mathrm{L}$. in relation to body size and condition factor. Fish. Res., 19: 193-204. https://doi. org/10.1016/0165-7836(94)90038-8

Satpathy, B.B., D. Mukherjee and A.K. Ray. 2003. Effect of dietary protein and lipid levels on growth, feed conversion and body composition in rohu, Labeo rohita (Ham.), fingerlings. Aquacult. Nutr., 9: 17-24. https://doi. org/10.1046/j.1365-2095.2003.00223.x

Shehawy, S.M.E, A.A. Gab-Alla. and H.M.A. Mutwally. 2016. Proximate and elemental composition of important fish species in Makkah central fish market, Saudi Arabia. Food Nutr. Sci., 07: 429- 439. https://doi. org/10.4236/fns.2016.76044

Ugwu,L.L.C.,B.O.Mgbenka,B.S. Valdon and C.D. Nwani. 2007. Factors on proximate composition of juvenile and yearlings of Heterobranhcus bidorsalis exposed to graded concentrations of bonny-light crude oil. J. Fish. Int., 2(3): 234241.

Wootton, R.J., 1990. Ecology of teleost fishes. Chapman and Hall, London. pp. 404. https:// doi.org/10.1007/978-94-009-0829-1

Yousaf, M., A. Salam and M. Naeem. 2011. Body composition of freshwater Wallago attu in relation to body size, condition factor and sex from southern Punjab, Pakistan. Afr. J. Biotechnol. 10: 4265-4268.

Zar, J.H., 1996. Biostatistical analysis. PrenticeHall, New Jersey. 